\title{
LA LEY MUNICIPAL DE BASILIPO
}

Four new bronze fragments (published by Julián González in SDHI 49, 1983, pp. 395 399 ) of the municipal charter of Basilipo (near Hispalis, in the Baetica province) are here settled, altogether with other two already known fragments of the same inscription, in their place (chapters 64-67) into the lex Flauia municipalis. The same legal chapters are entirely given by the copy of Malaca and the recently discovered copy of Irni. By a comparative study of the whole text distribution by tables, columns and lines in the different copies of this lex data we can see how each municipe used to arrange the same legal text according to own taste and material possibilities. The concentration of those conserved municipal copies into a limited country of the Baetica shows the local emulation and the pride of the municipes in order to become Roman citizens per honorem, few years after ius Latii was given to Spain by Vespasian.

Julián González ha publicado ( $S D H I, 1983$, p. 395 ss.) cuatro nuevos fragmentos de bronce que pertenecen evidentemente a la ley municipal de Basilipo *. El nombre del municipio aparece tan sólo -incompleto, pero parece cierto- en el último fragmento: Bas]ilipon[ensis. Este municipio se suele localizar hoy en el Cerro del Cincho, dentro del término de Carmona (Sevilla). No aparece mencionado por Plinio, pero sí en el Itinerario Antoniniano, aunque con algún posible desajuste en la distancia miliaria; Julián González rectifica esa identificación usual del municipio, habida cuenta del hallazgo de los nuevos fragmentos en el Rancho de la Estaca, cerca de Puebla de Cazalla, en la misma provincia de Sevilla. No entramos ahora en este problema topográfico, teniendo en cuenta que, aun admitiendo el posible error del Itinerario Antoniniano, la conservación de bronces legales no siempre es en la

* Redactado ya este artículo, el mismo J. González ha tenido la gentileza de comunicarme el original de otro que se publicará en la $Z P E$, en el que se presenta también la reconstrucción de conjunto del Bronce de Basilipo; las pequef́as diferencias en la distribución de las líneas es siempre superable, pero quizá no muy relevante, pues es conjetural. 
misma localidad a que éstos originariamente pertenecían; los bronces de Salpensa y Málaga, por ejemplo, aparecieron juntos, fuera de su lugar de origen.

Estos cuatro nuevos fragmentos, como acertadamente ha visto Julián González, deben unirse a los otros dos que había juntado yo en EMERITA, 1964, p. 103: uno de ellos era entonces nuevo y se decía proceder de El Rubio, es decir, el mismo lugar de la provincia sevillana en que habían aparecido los fragmentos de la ley colonial de Vrso que, a instancias de mi maestro Juan de M. Carriazo, había publicado yo en EMERITA, 1941, p. 138 (cf. EJER, p. 239); el otro era conocido de antiguo, y se daba como exportado y, al parecer, perdido, en Francia: era el núm. 31 de las Fontes de Bruns, y que yo mismo recogía en mi EJER con el núm. 10.

Estos seis fragmentos pertenecen a una misma tabla y deben ordenarse conjuntamente, pues corresponden a la secuencia de los capítulos 64-67 de la ley municipal que sirvió de modelo a todas las copias que tenemos de esa ley, de distintos municipios de la Bética, a las que me refiero seguidamente, y deben numerarse por el orden del mismo texto a que corresponden. Así, los cuatro nuevos fragmentos (González [SDHI, 1983]: I-IV), quedan numerados como $a, b, c$ y $f$, ya que entre los dos últimos deben intercalarse los dos de EMERITA, 1964: el entonces nuevo $(d)$ y el perdido en Francia $(e)$. En realidad, $e$ y $f$ son paralelos (en la parte final de nuestra reconstrucción), pero el $f$, aunque empieza una línea antes que $d$, está a la derecha de éste, separado por unas pocas letras perdidas de las mismas líneas, y por eso me parece que debe colocarse como último. Es en este último frag. $f$ donde aparece el nombre del municipio.

El estudio de las leyes municipales de la Bética solía hacerse por separado, y tomando como principales la tabla de Salpensa (EJER, núm. 8), con dos columnas, y la de Málaga (EJER, núm. 9), con cinco columnas. Pero hoy podemos ver, no sólo que estas tablas y los otros fragmentos menores siguen un modelo único, lo que ya podía sospecharse antes, sino que tenemos como copia mejor conservada $\mathrm{y}$, por tanto, como principal, respecto a la cual todas las demás resultan accesorias, la nueva y todavía inédita del municipio Irnitano, hallada hace ya unos años en El Saucejo, cerca siempre de Sevilla. Tampoco de este municipio teníamos datos suficientes. Podemos conjeturar que su nombre era Irni, y que era un municipio de importancia mínima, a pesar de que su ley nos resulta ahora el ejemplar principal.

Así, pues, por orden de extensión, contamos ahora con estas copias de la ley municipal común: Irnitana, Malacitana, Salpensana, esta 
Basiliponense, y la Italicense (EJER, núm. 11: un pequeño fragmento del final de la ley [que Alicia Canto atribuye ahora a Cortegana y no a Itálica, problema en el que no entro aquí]).

Gracias a la Irnitana hemos podido ver que hubo una ley fundamental de Augusto (poco posterior al 17 a. C.), dada para los municipios de Italia, y hay luego un texto adaptado (aunque no debemos excluir la posibilidad de sucesivas reformas) de aquella ley a los municipios de provincias, y, en concreto, a los de España, que habían recibido el ius Latii concedido por Vespasiano, es decir, la facultad de acceso a la ciudadanía romana por los honores municipales. Esta ley adaptada es la que se copia, por todos esos municipios que hemos mencionado, en la época de Domiciano, y por eso creo que podemos hablar de una lex Flauia municipalis como texto muy reformado de la lex Iulia municipalis, que, como hemos dicho, resulta ser de Augusto y no de César. No vamos a hablar hoy de este nuevo texto de excepcional importancia, sino que se trata tan sólo de presentar el texto de los capítulos 64-67 de la ley Flavia tal como debía de reproducirse en la copia de Basilipo, colocando los seis fragmentos conservados en el lugar que les corresponde.

Señalamos al margen los números de las líneas de los seis fragmentos $(a-f)$, pero indicamos también (en la parte interior del mismo margen) la numeración seguida (entre paréntesis y de cinco en cinco) de las líneas de todo el texto, no según la copia malacitana, sino según la irnitana, en cuya tabla VIII, a lo largo de sus dos últimas columnas $(B$ y $C$ ) se extiende el texto de estos cuatro capítulos. Pero hay que tener en cuenta que todo este mismo texto se reproduce igualmente en la tabla Malacitana, en sus dos últimas columnas. Tenemos así, en este lugar del texto legal, la coincidencia de tres copias de distintos municipios, lo que, por ahora, no se vuelve a dar en ningún otro lugar de aquel texto, donde, a lo más, hay coincidencia de dos copias.

Por lo que a la ley Irnitana se refiere, la correspondencia de nuestros seis fragmentos con las líneas de aquella ley es la siguiente:

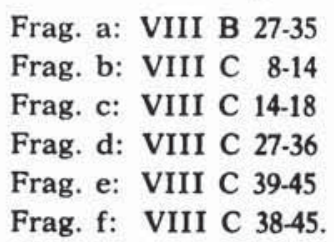

Después de presentar el texto, haremos algunas breves observaciones sobre la posible disposición de esta ley de Basilipo, en comparación con las otras cinco conocidas. 
(VIII, B 14-15)

[R De obligatione praedum et praediorum / cognito-] [rumque] /

[Quicumque in municipio Flauio Basiliponensi in commu/ne] [LXIV] [municipum eiius municipii praedes facti sunt erunt,] / [quaeque praedia accepta sunt erunt, quique eorum] / [praediorum cognitores facti sunt erunt, ii omnes] /

(20) [et quae cuiiusque eorum tum erunt, cum praes cogni-] / [torue factus est erit, quaeque postea esse, cum ii ob/li-] [gati esse coeperunt coeperint, qui eorum soluti li/be-] [ratique non sunt non erunt, aut non sine d.m. sunt / erunt,]

(25) [eaque omnia quae eorum soluta liberataque / non sunt] [non erunt, aut non sine d.m. sunt erunt, in com/mune mu-] [nicipum eiius municipii, item obligati ob/ligataque]

Frag. a 1 [sunto ut]i ii eaue p.R. [obligati obliga/taue essent, si] 2 [aput e]os qui Romae aerario praee[ssent, ii / praedes iiq. cog-] 3 (30) [nitores] facti aeque praedia sub[di/ta subsignata obliga-] [taue esse]nt. Eosque praedes eaque / [praedia eosque cogni-] [tores, si qui]t eorum in qua〈e / cognitores facti erunt] [ita non erit q]ui quaeue so/luti libe[rati soluta libe-] 7 [rataque non sunt] non / erunt, aut n[on sine d.m. sunt erunt,] 8 (35) [IIIuiris qui ibi / iur]i d. p[raeerunt, ambobus alteriue eorum ex] [decurionum / conscriptorumue decreto, quod decretum cum] [eo/rum partes tertiae non minus quam duae adessent] / [factum erit, uendere legemque eis uendundis dicere / ius]

(40) [potestasque esto, dum eam legem iis rebus uendun/dis] [dicant quam legem eos qui Romae aerario praeerunt e] [lege praediatoria praedibus praedisque uen/dundis di-] [cere oporteret, aut, si lege praediatoria empto/rem] [non inuenerit, quam legem in uacuom uen/dendis di-]

(45) [cere oporteret, et dum ita legem dicant uti / pecunia] [in foro municipii Flaui Basiliponensis refera/tur] [luatur soluatur. Quaeque lex ita dicta erit iusta / ra-] [taque esto.] /

(VIII, C 1)

[R Ut ius dicatur e lege dicta praedibus et prae-] [dis uen/dundis.]

[Quos praedes quaeque praedia quosque cognitores II uir./ mu-]

[LXV] [nicipi Flaui Basiliponensis h.l. uendiderint, de iis / ]

(5) [quicumque i.d. praeerit ad quem d.e.r. in ius aditum erit ita] [ius / dicito iudiciaq. dato, uti ei qui eos praedes cogni-] [tores ea / praedia mercati erunt praedes socii heredesq.]

Frag. b 1 [eorum / iique ad quos e.r. pertinebit de] is rebus [agere] 2 [easque res pete/re persequi recte pos]sint. 
(VIII, C 1)

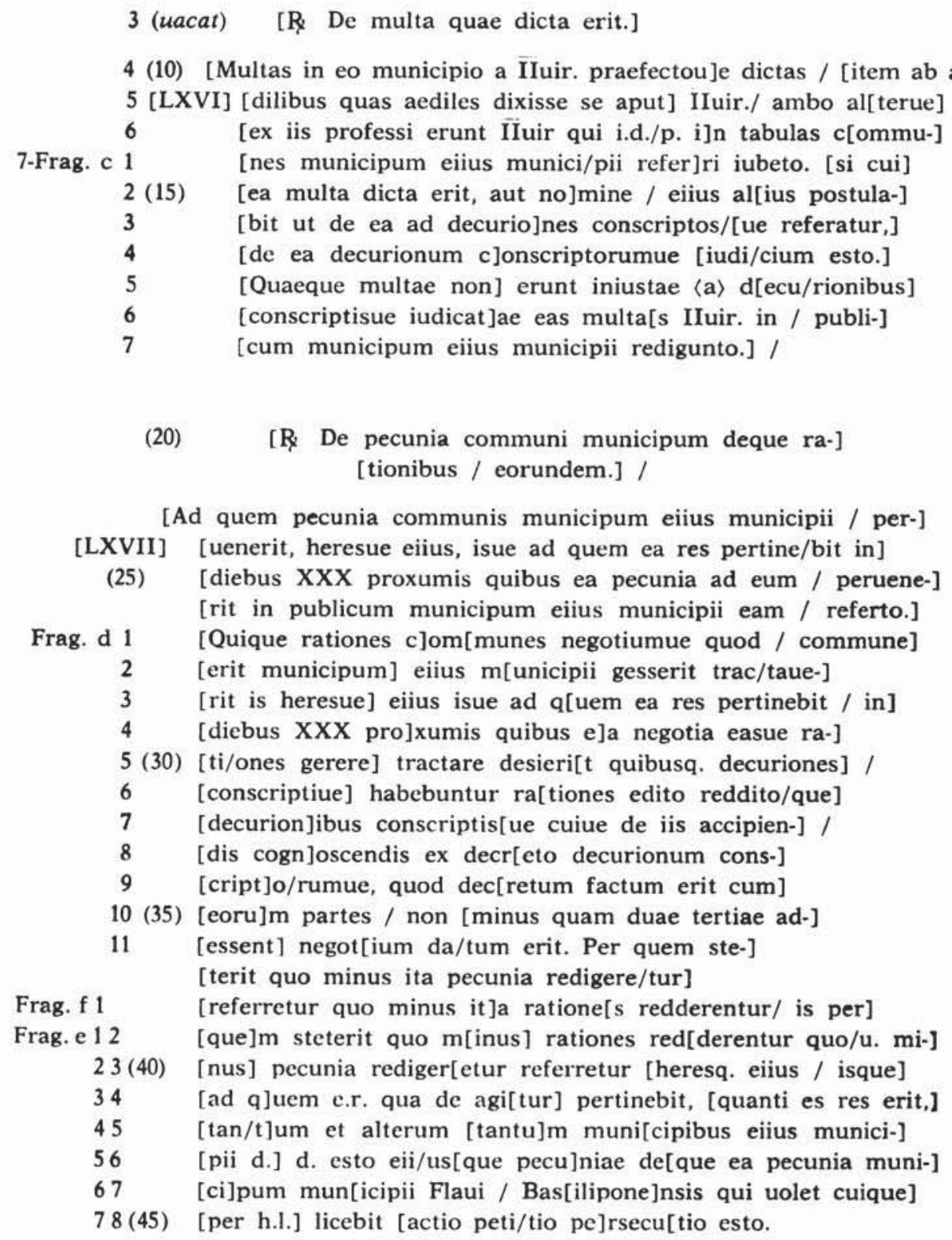

Las diversas copias de la lex Flauia municipalis quizá fueron obtenidas de un mismo modelo manuscrito, que, en poder del gobernador provincial, al que había llegado desde Roma, debía de prestarse a los municipios a fin de sacar en cada uno de ellos la correspondiente copia. 
Es posible que la gestión de traer el manuscrito fuera llevada a cabo por un legatus del municipio, pues, al final de la Irnitana, aparecen los nombres de dos Caecilii, uno duumuir y el otro legatus, como personas encargadas de la publicación del texto en bronce; que la embajada fuera a la capital de la Bética, Córdoba, donde supongo que debería hallarse el manuscrito, en poder del praeses de la provincia, y no a Roma, me parece lo más probable; así como que la recitatio de una epistula de Domiciano, que figura al final del texto irnitano, debió de consistir en una lectura ante la curia decurional de Irni, y no, en cambio, en el palacio del gobernador provincial por cuyas manos es lo más probable que hubiera pasado la carta llegada de Roma (aunque había sido dictada por Domiciano en su finca de recreo de Circeo).

Variaciones entre las copias eran siempre posibles, como podemos comprobar, a pesar de que el modelo original usado por los distintos copistas fuera el mismo, probablemente un uolumen en papiro, con una larga serie de columnas, desde luego de menor contenido que el de las de los bronces. La misma distribución de las tablas, con más o menos columnas, dependería de circunstancias materiales como, ante todo, las posibilidades de la forja de bronce y del gusto y costumbre del encargado de la grabación. Pero podemos observar que las columnas suelen ser más largas, es decir, de mayor número de líneas, cuando éstas son más cortas, es decir, de menos letras, por lo que la diferencia entre columnas, entre las diversas copias, no es excesivamente apreciable, salvo en Itálica. Al mismo tiempo vemos que, cuando las columnas son más estrechas, aumenta el número de ellas en cada tabla. El siguiente cuadro puede dar una idea de la proporción y variaciones entre las distintas copias:

$\begin{array}{lccccc}\quad \text { Copia } & \text { Núm. de tablas } & \begin{array}{c}\text { Columnas } \\ \text { por tabla }\end{array} & \begin{array}{c}\text { Lineas por } \\ \text { columna }( \pm)\end{array} & \begin{array}{c}\text { Letras por } \\ \text { linea }( \pm)\end{array} & \begin{array}{c}\text { Letras por } \\ \text { columna }( \pm)\end{array} \\ \text { Malacitana } & 6 & 5 & 70 & 32 & 2.240 \\ \text { Irnitana } & 10 & 3 & 53 & 43 & 2.289 \\ \text { Salpensana } & 15(?) & 2 & 58 & 44 & 2.552 \\ \text { Italicense } & 8(?) & 2(?) & 62 & 69 & 4.278\end{array}$

Como puede observarse, el número de letras por columna viene a ser muy parecida en las tres primeras copias (Irnitana, Malacitana y Salpensana). La diferencia no llega a ser, en total, la de una columna más o menos; esto, a pesar de que unas 24 líneas de la última columna Irnitana está ocupada por aditamentos que probablemente faltaban en las otras copias, como sabemos que ocurría en la Italicense, cuyo final, precisamente, es lo que se conserva. Podemos partir así de la hipótesis 
de que el número de 30 columnas de la Irnitana era el mismo para la Malacitana y la Salpensana; sólo que con distinto reparto de estas 30 columnas en cada tabla. En cambio, la mayor cantidad de letras por columna en la Italicense obliga a conjeturar un menor número total de columnas (16?).

¿Qué puede deducirse de la comparación con la ley Basiliponense?

Tenemos aquí una secuencia de cuatro capítulos (64-67), con un total de 78 líneas, el mismo número que en la copia Irnitana, donde ocupan aquéllas, como hemos dicho, parte de dos columnas; pero no sabemos cuántas líneas tenía cada columna de la copia Basiliponense.

No sería imposible pensar en columnas muy largas - de, por lo menos, 80 líneas, pues el último fragmento no conserva restos del margen inferior-, aún más largas que las de la copia Malacitana, de 70 líneas o poco más; pero lo más probable es que estos cuatro capítulos no ocuparan una sola columna, sino que se extendieran por dos columnas, aunque resulta difícil decir dónde podía terminar la primera y empezar la segunda. Una continuidad indubitable hay entre los frags. $b$ y $c$, puesto que ambos conservan partes de una misma letra - la $O$ de iubeto en la lín. VIII, C 14- Luego, es evidente que los frags. $e$ y $f$ son de las mismas líneas, y la separación respecto al frag. $d$ por una sola línea totalmente perdida, obliga a pensar que estos tres fragmentos pertenecían a la misma columna. En cambio, nada impediría que los dos fragmentos anteriores, o sólo el $a$, más distanciado del resto, pertenecieran a una columna anterior. Pensar que los seis fragmentos sean de la misma columna, y que sólo las aproximadamente diez líneas, antes del frag. $a$, sean de la columna anterior, obligaría a conjeturar unas columnas tan largas como las de la copia Malacitana, aunque esto no sería imposible. Un cambio apreciable en la longitud de las líneas, de manera que pudiera verse un tránsito de una columna a otra, no se da en nuestro caso.

Como es sabido, en estos textos, el cálculo de letras por línea no puede ser nunca del todo seguro. Aunque la diferencia de espacio que ocupan las distintas letras - desde las anchas $M M$ hasta las delgadas letras de un solo trazo vertical $(I, E, T, L)$ - puede ser compensable en una línea algo larga, la inseguridad del cálculo puede deberse a distintas causas:

i) Un mismo copista puede no mantener durante todo su trabajo una regularidad perfecta, y podemos ver que, aparte la irregularidad de los puntos de separación - de los que hemos prescindido en nuestra transcripción-, la hay en la misma separación de palabras, en el aprovechamiento o no del "uacat» de líneas finales de capítulo para alojar 
las rúbricas de los siguientes, y otros detalles que pueden alargar o encoger el texto. Esto, aparte la deliberada reducción y apretamiento de algunos trozos que se puede observar en las últimas tablas de Urso y de Irni.

ii) También hay oscilaciones, en un mismo texto, en razón de las frases abreviadas o no. Así, p. ej., en nuestro frag. $a$, lín. 1 leemos $p$ (opulo) $R$ (omano), abreviado, como también en la copia malacitana, cuando en la Irnitana está sin abreviar; en el mismo fragmento, lín. 8, leemos iur]i d(icundo) p[raeerunt, expresión que aparece más o menos abreviada según las copias; $\mathrm{y}$, en esta misma inscripción (frag. $b$, lín. 6) hemos de suponer $i$. $d$. p p. totalmente abreviado; lo mismo ocurre con e(a) $r(e s)$ y otras frases usuales. Así también, $\bar{I} u i r(u m)$ (frag. $b$, lín. 5) aparece otras veces sin abreviación de la desinencia, e incluso con todas las letras (duumuir), lo que supone una notable diferencia. Las enclíticas $-u(e)$ y $-q(u e)$ pueden aparecer unas veces en forma abreviada y otras no; como ocurre con $d($ olo $) m($ alo), d(are) d(amnas) e(sto), etc. En nuestra transcripción hemos prescindido de resolver las abreviaciones usuales de este tipo, con el fin de no sobrecabalar el encaje de las líneas.

iii) Luego, aunque en mínima medida para el cálculo de letras, hay que contar con las oscilaciones ortográficas, incluso en un mismo copista; por ejemplo, la duplicación de la $I$ intervocálica (eiius-eius), del genitivo (municipii), o en el pronombre iis - is, etc.; en la Irnitana, el copista tiende a no duplicar, pero en la Basiliponense, como en la Malacitana, sí. De estas oscilaciones, así como de otras que casi no afectan a la extensión de las líneas (como $t$ por $d$ : at-ad, aput-apud, o proximus - proxumus), se puede prescindir en una transcripción como la nuestra.

iv) Luego, como probablemente el copista no entendía lo que escribía, que no siempre es fácil de entender, hay que contar con errores varios de transcripción (como en el frag. $d$, lín. 8, donde se lee ea en vez de ex), y para nuestro interés del cálculo de extensión de líneas (para poder proponer suplementos), sobre todo con errores de omisión (como la omisión de $\langle a\rangle$ en frag. c, lín. 5) o de repetición de palabras; no en nuestros fragmentos, pero sí, p. ej., en la Malacitana, líns. 45-46 (en el lugar correspondiente a la lín. 43), el copista volvió a escribir, tras Malacitani, más de una línea sobrante: eius ea pecunia municipum municipii Flaui Malacitani.

Se comprende, pues, que un cálculo de letras por línea no puede ser muy exacto. En nuestro texto, desde la lín. 33 a la 37 da la impresión 
de que las líneas se acortan, pero precisamente ahí los restos del frag. $d$ nos dan una pauta segura para la reconstrucción de esas líneas, y, por lo demás, a partir de la 38, parece recuperarse la longitud ordinaria de las líneas. En algún caso más llamativo, como es el de la lín. 37, hay que sospechar que hubo alguna repetición indebida por parte del copista, sea en esta misma línea o en la parte final de la anterior. Acaso se podría esperar una mejor distribución corriendo el frag. $d$ un poco más a la derecha, pero no se conseguiría gran cosa con ello, pues, como digo, los restos de las últimas líneas de este fragmento impiden recorrer el texto para completar mejor esas pocas líneas más cortas.

Así, pues, tampoco por un cambio en la extensión de las líneas puede apreciarse el paso de una columna a otra.

Sea como sea, lo que sí parece seguro, como hemos dicho, es que los seis fragmentos pertenecen a una misma tabla; esto, a pesar de que no fueron hallados juntos todos ellos.

Por último, ignoramos también cuántas columnas podían tener las tablas de la ley de Basilipo: si tres, como la de Irni, cinco, como la Malacitana, o bien dos, como, al menos en la que tenemos, había en la Salpensana; ni siquiera si todas las tablas de esa ley tenían el mismo número de columnas, pues, como vemos en la ley de Vrso, podía haber un conjunto simétrico de tablas desiguales. En consecuencia, no sabiendo el número de columnas de cada tabla, ni su regularidad o no, ni la longitud de las columnas, tampoco podemos saber el número de tablas de esa misma ley. Vemos que las líneas eran aproximadamente de la misma extensión que las de Irni, pero sólo por suposición podemos pensar que tendría diez tablas como la Irnitana, con tres columnas cada una, y un número de líneas que oscilase alrededor de 52 por cada columna. Aceptada esta suposición, por la simple comparación con la copia irnitana, podría pensarse que el frag. a pertenecería, como el texto correspondiente de Irni, a la segunda columna de la tabla VIII y los otros cinco $(b-f)$ a la tercera.

En conjunto, aunque sin gran seguridad, la disposición de la ley de Basilipo debía de ser más similar a la Irnitana y Salpensana, que a la Malacitana, con sus líneas más cortas, y, sobre todo, a la Italicense, con sus líneas muy largas.

En este campo de las leyes municipales de la Bética siempre cabe esperar que se produzcan nuevos hallazgos, incluso de tablas o fragmentos de las copias ya conocidas.

En fin, cabría preguntarse por qué sólo aparecen estos restos de la lex Flauia municipalis en esa zona tan reducida de la Bética. No es 
que falten otros restos mínimos en la Citerior (Ampurias y Clunia), pero no parecen coincidir con el modelo flavio, y no hay que excluir que estos y otros restos que puedan aparecer todavía pertenezcan al antiguo modelo de la lex Iulia municipalis.

Aunque el ius Latii, que determinó ese afán por exhibir la condición municipal -y nada mejor para ello que la ostentación de una larga ley en bronce-, fue concedido a todos los municipios de Hispania, la verdad es que el florecimiento de la vida municipal fue sobre todo notable en la Bética. Con todo, esa concentración de restos de la lex Flauia en una zona tan reducida dentro de la Bética, así como el hecho de la contemporaneidad de los mismos, de fines del siglo I d. C., inducen a pensar que esa concentración puede deberse a otras circunstancias, como el hecho de una mayor o menor conservación de los bronces, pero quizá también a que pudo darse precisamente en esa pequeña zona una cierta emulación entre municipios vecinos, muchos de ellos tan insignificantes como Irni, Salpensa y Basilipo; en una palabra: moda, pero una moda escasamente extendida, y que duró poco tiempo, porque la verdad es que, al cabo de no muchos años, la ciudadanía romana se hallaba ya tan difundida, no siempre legalmente -incluso en esa misma zona de la Bética, como hace sospechar ya la epístola de Domiciano, para subsanar matrimonios irregulares, que seguía al texto de la ley Irnitana-, que dejó de tener interés la exhibición de la misma, y cesó aquel afán de tener una prueba visible de la municipalidad por la ostentación de leyes en bronce. Porque no hay que perder de vista que esa ley municipal dada por Augusto, incluso después de su adaptación flavia a los municipios de la Bética, acabaría por no observarse muy rigurosamente, y que, como suele ocurrir con muchas leyes, era más una forma de propaganda oficial que de régimen realmente necesario y vigente. Por lo demás, sabemos que la decadencia de las autonomías municipales, que aparece como crisis clamorosa en siglos posteriores, realmente se había iniciado ya en el siglo II. El mismo esplendor aparente de la época de los Antoninos correspondía a un profundo deterioro, causado, al menos en parte, por la pretensión de mantener un rango público para el que no existían las reservas necesarias. Una pequeña muestra de esto tenemos en la evidencia del abuso escandaloso, no exclusivo de Hispania, de las embajadas municipales con gastos pagados, que Antonino Pío hubo de reprimir, con más energía quizá que éxito, pero un abuso contra el cual ya había intentado oponerse Adriano. Inevitablemente, este deterioro de una autonomía municipal responsable hubo de producir un mayor intervencionismo del gober- 
nador de la provincia, del que se podía quizás esperar mayor seriedad en la administración de la pecunia communis, tema muy importante de la ley municipal.

Sobre la interpretación, en concreto, de esos capítulos 64-67 trato nuevamente en mi estudio sobre la lex Flauia municipalis (Roma, en prensa).

Alvaro d'ORS 